\title{
NOVEL MECHANISM OF BIQUADRATIC COUPLING IN MAGNETIC SUPERLATTICES
}

\author{
W. GRUHN \\ Institute of Physics, Pedagogical University \\ Al. Armii Krajowej 13/15, 42-201 Częstochowa, Poland
}

\begin{abstract}
Using the modified total energy approach of Bruno, we prove that for the transition metal or rare-earth superlattices the additional scattering of free electrons on magnetic ion multipole moment gives rise to ferroquadrupolar biquadratic coupling between magnetic layers. We show that in this novel, intrinsic mechanism, the anisotropy of the magnetoresistivity should manifest itself.
\end{abstract}

PACS numbers: $75.70 . \mathrm{Cn}$

The exchange coupling between ferromagnetic layers separated by a thin non-magnetic, metallic spacer, is currently an object of intense investigation. The recently discovered, $\pi / 2$ coupling, between magnetizations of the neighbouring magnetic layers [1] added new interest to the problem [2]. Although the dominating mechanism for this exchange interaction has been unambiguously associated with the Ruderman-Kittel-Kasuya-Yoshida (RKKY) interaction [3] the specific mechanisms responsible for the entire coupling are not yet fully understood. There are basically two strategies which have been used theoretically to study the interlayer magnetic coupling: (i) total energy calculations and (ii) perturbative models. Most applications of the latter approach follow earlier studies on the coupling between magnetic impurities in a host metal. The coupling of the ionic spin $S_{n}$ with the itinerant electron spin $\sigma$ is usually taken as the contact interaction [4]:

$$
H_{\mathrm{ex}}=-2 J S_{n} \sigma \delta(r) \text {. }
$$

The resulting interaction between magnetic ions is the RKKY-reminiscent, bilinear in ionic spin operators, coupling. Very recently, within the perturbative approach, there has been derived a new form of quadrupolar coupling of the magnetic ions across the nonmagnetic, metallic spacer [5]. In case of non-s magnetic ion state, along with the scattering due to dipolar contact interaction, there appears also scattering of conduction electrons of quadrupolar moments. Kondo [6] has proved that the interaction $V_{\mathrm{qc}}(k)$ between conducting electrons and the quadrupoles is given by

$V_{\mathrm{qc}}=\frac{D}{k_{\mathrm{F}}{ }^{2}} \sum_{k+k-\sigma^{+\sigma^{-}}}\left[\left(S_{n} \cdot k^{+}\right)\left(S_{n} \cdot k^{-}\right)-\frac{1}{3} S(S+1) k^{+} \cdot k^{-}\right] c_{k+\sigma^{+}}^{+} c_{k-\sigma^{-}}$, 
where $k^{+}, \sigma^{+}, k^{-}$, and $\sigma^{-}$denote the spin and wave vector of the impact and the scattered electron, respectively. $D$ is a constant which determines the strength of the scattering potential. Using the scattering potential (2), within perturbative approach, we showed that the interaction between quadrupole moments of magnetic ions $\left(Q_{i}\right.$ and $\left.Q_{j}\right)$ is given by [5]:

$$
H\left(R_{i j}\right)=-\sum_{\alpha \beta \gamma \delta} Q_{\alpha}^{\beta} Q_{\gamma}^{\delta} \Lambda_{\alpha \beta}^{\gamma \delta}\left(\left|R_{i}-R_{j}\right|\right),
$$

where the $Q_{\alpha}^{\beta}$ denote the respective components of ionic quadrupole moment

$$
Q_{\alpha}^{\beta}=\left[S_{n}^{\alpha} S_{n}^{\beta}-\frac{1}{3} S(S+1) \delta_{\alpha \beta}\right] .
$$

Similarly to the case of bilinear spin-spin exchange, the interaction (3) favours the ferromagnetic (ferroquadrupolar) coupling [5]. The effective coupling between the magnetic layers in a superlattice, may be obtained by summation of coupling contributions from all moments within magnetic sheets [7]. This approach is justified, provided that the interionic coupling is isotropic, which does not hold in the superlattice systems [8]. Moreover, in spite of the success of the perturbative methods in explaining the coupling between magnetic impurities in transition metals (TM) or noble metals, one might be skeptical about the results of a perturbative calculation of the coupling between two sheets of spins, especially when the spacer layer has a propensity to be magnetic [10].

The aim of the paper is to extend the mechanism of interionic quadrupole interaction in a layered system discussed in Ref. [5] on the superlattice. In view of the discussion above, one should rely on total energy calculation. In the following, we will modify the quantum well electron confinement approach of Bruno [3] to describe the coupling due to the potential scattering (2). The model system consists of a metallic spacer layer (paramagnetic), sandwiched between two potential perturbations of height $V^{A}$ and $V^{B}$, generated by, adjacent to the spacer, magnetic layers. The effective coupling between magnetic sheets across the spacer, is associated with the multiple internal reflections of free electrons at the interfaces. The resulting "quantum interference" produces interlayer coupling, which oscillates with a period directly related to the geometry of the system. As it was shown by Bruno [3], the energy change due to the quantum interferences within spacer is given by

$$
\Delta E=\frac{2}{\pi} \int_{\infty}^{\epsilon_{F}} \ln \left|1-r_{A} r_{B} \mathrm{e}^{2 \mathrm{i} k_{\perp} L}\right| \mathrm{d} \epsilon \approx \frac{1}{\pi^{3}} \int \mathrm{d}^{2} k_{\|} \int_{-\infty}^{\epsilon_{F}} r_{A} r_{B} \mathrm{e}^{2 \mathrm{i} k_{\perp} L} \mathrm{~d} \epsilon,
$$

where $r_{A}$ and $r_{B}$ are the reflection amplitudes at both interfaces, $L$ is the spacer thickness $L=(N+1) d$, with $d$ being the thickness of one atomic plane in the spacer, while $k_{\perp}$ denotes the perpendicular to interface component of the impact electron wave vector.

Let us assume that the magnetizations of the ferromagnetic layers are at angle $\theta$ with respect to each other and determine the coupling energy $\Delta E(5)$ as the function of $\theta$. In the zeroth approximation, the reflection amplitude at the interface $A$ is given by [9]:

$r_{A}=A\left(k_{\perp}^{+}, k_{\perp}^{-}\right)\left\langle k^{-}\left|V^{A}\right| k^{+}\right\rangle=A\left(k_{\perp}^{+}, k_{\perp}^{-}\right)\left\langle k^{-}\left|V_{0}^{A}+V_{\mathrm{qc}}^{A}\right| k^{+}\right\rangle=r_{A}^{0}+r_{A}^{\mathrm{qc}}$, 
where

$$
r_{A}^{0}=A\left(k_{\perp}^{+}, k_{\perp}^{-}\right)\left\langle k^{-}\left|V_{0}^{A}\right| k^{+}\right\rangle=A\left(k_{z}\right)\left\langle k^{-}\left|V_{0}^{A}\right| k^{+}\right\rangle
$$

gives us the contribution from isotropic potential as well as from exchange contact interaction given by (1). The $r_{a}^{\text {qc }}$ is the reflection amplitude due to the electron-quadrupole scattering (2). Let us focus our attention on this term. In Eq. (7) we should account that wave vectors of the incident $k^{+}$and reflected $k^{-}$ electron fulfil the relation $k_{\perp}^{+}=-k_{\perp}^{-}$. To account the effect of magnetization canting one must note that the scattering due to the quadrupolar potential (2) does not depend on the spin of impact electron. This means that the electron reflection amplitudes are equal, i.e. $r_{A B}^{\mathrm{qc} \uparrow}=r_{A B}^{\mathrm{qc} \downarrow}$. Thus, we must modify the approach of Bruno [3], who exploits the spin dependence of the reflection amplitudes. For the sake of simplicity, in the further considerations, we will replace the spin operators $S_{i}$ in Eq. (2) by their average, i.e. the layer magnetization $\boldsymbol{M}$. Provided that the parallel to interface component of the impact electron wave vector makes angle $\phi$ with the in-plane magnetization of the $A$-th layer, in view of $\mathrm{Eq}$. (2), we can write the reflection amplitudes as

$$
r_{A}^{\mathrm{qc}}(\phi) \approx\left\langle k^{-}\left|V_{\mathrm{qc}}^{A}\right| k^{+}\right\rangle=C_{3}\left[C_{1} \cos ^{2} \phi+C_{2}\right]
$$

where

$$
\begin{aligned}
& C_{1}=M^{2} k_{\|}^{2}, \quad C_{2}=\frac{1}{3} S(S+1)\left(2 k_{\|}^{2}-\frac{2 m}{\hbar^{2}} \epsilon\right), \\
& C_{3}=\frac{-a^{2}}{\frac{\hbar^{4}}{m^{2}}\left(\frac{2 m}{\hbar^{2}} \epsilon-k_{\|}^{2}\right)} .
\end{aligned}
$$

Similarly, assuming that magnetization of the second layer is parallel to the layer and makes angle $\theta$ with the magnetization of the other layer, we can write

$$
r_{B}^{\mathrm{qc}}(\phi \pm \theta) \approx\left\langle k^{-}\left|V_{\mathrm{qc}}^{B}\right| k^{+}\right\rangle=C_{3}\left[C_{1} \cos ^{2}(\phi \pm \theta)+C_{2}\right] \text {. }
$$

Integration over $k_{\|}$in Eq. (5) performed in polar coordinates $\left(\left|k_{\|}\right|, \phi\right)$ gives us

$$
\int_{0}^{2 \pi} \mathrm{d} \phi r_{A} r_{B}(\phi, \theta) \approx \int_{0}^{2 \pi} \mathrm{d} \phi C_{3}^{2}\left[C_{1} \cos ^{2}(\phi \pm \theta)+C_{2}\right]\left[C_{1} \cos ^{2} \phi+C_{2}\right] .
$$

Having the result (12), we can find that the energy change $\Delta E$ given by formula (5) depends on $\theta$ as

$$
\Delta E(\theta)=\Delta E\left(\cos ^{2} \theta\right) \approx J_{0}+J_{2} \cos ^{2} \theta+\ldots
$$

where the coefficient $J_{2}$, which determines the dependence of total energy on $\cos ^{2} \theta$, can be interpreted as the biquadratic exchange integral [3]. In view of Eqs. (9)-(13), $J_{2}$ is given by the following expression:

$$
J_{2}=\frac{D^{2}}{k_{\mathrm{F}}{ }^{4}} \frac{\pi}{2} M^{4} \int k_{\|}^{4} \mathrm{~d} k_{\|} \int \mathrm{d} \epsilon \frac{-a^{2}}{\frac{\hbar^{4}}{m^{2}}\left(\frac{2 m}{\hbar^{2}} \epsilon-k_{\|}^{2}\right)} .
$$

In view of the result (13), we can claim that within the total energy calculation method we proved that the scattering potential (2) is the source of biquadratic exchange between magnetic layers.

Earlier approach to the problem under consideration was based on the perturbative method [5]. The resulting interionic quadrupole-interaction consists of up 
to six independent coupling parameters, which describe coupling between respective components of the ionic quadrupole moments. Application of these results to determination of the effective biquadratic interaction between adjacent to spacer magnetic layers, requires summation of coupling contributions from all moments within magnetic sheets. Thus, the determination of the parameters of biquadratic exchange which relies on perturbative approach suffers many difficulties.

In order to circumvent these problems, we devised a modification of the "quantum interferences" method of Bruno [3], which allows us to calculate the energy of the effective coupling as a function of angle $\theta$ between magnetizations on the neighbouring magnetic sheets. The approach presented above gives a precise, quantitative relationship, between strength of the quadrupolar scattering potential and the biquadratic exchange integral $J_{2}$. In view of the general properties of the quantum interferences due to the electron confinement within spacer, the effective biquadratic exchange integral oscillates with the spacer thickness [3]. Finally, let us mention that in the bulk systems the potential (2) is the source of the anisotropy of magnetoresistivity [11], i.e. the effect often observed in the magnetic superlattices.

\section{Acknowledgments}

I am grateful to Professor Z. Bak for stimulating discussions. Financial support from the Committee for Scientific Research is gratefully acknowledged.

\section{References}

[1] B. Heinrich, J.F. Cochran, M. Kowalewski, J. Kirschner, Z. Celiński, A.S. Arrott, K. Myrtle, Phys. Rev. B 44, 9348 (1991).

[2] J.C. Slonczewski, Phys. Rev. Lett. 67, 3172 (1991).

[3] P. Bruno, Phys. Rev. B 52, 411 (1995).

[4] M.A. Ruderman, C. Kittel, Phys. Rev. 96, 99 (1954).

[5] W. Gruhn, Z. Bąk, R. Jaroszewicz, Acta Phys. Pol. A 89, 417 (1996).

[6] J. Kondo, Prog. Theor. Phys. 27, 772 (1962).

[7] W. Baltensperger, J.S. Helman, Appl. Phys. Lett. 57, 2954 (1990).

[8] Z. Bak, W. Gruhn, J. Magn. Magn. Mater. 131, 210 (1994).

[9] P. Bruno, J. Magn. Magn. Mater. 121, 248 (1993).

[10] D.M. Deaven, D.S. Roksar, M. Johnson, Phys. Rev. B 44, 5977 (1991).

[11] A. Friedrich, A. Fert, Phys. Rev. Lett. 33, 1214 (1977). 